\title{
Why Should Clinicians Publish?
}

\author{
Rijo Mathew Choorakuttil' \\ ${ }^{1}$ Research Action Group of AMMA Education Research Foundation, \\ AMMA Center for Preventive and Diagnostic Medicine, Kochi, \\ Kerala, India
}

\begin{abstract}
Address for correspondence Rijo Mathew Choorakuttil, MD, Research Action Group of AMMA Education Research Foundation, AMMA Center for Preventive and Diagnostic Medicine, Kochi 682306, Kerala, India (e-mail: erfamma@gmail.com).
\end{abstract}
Abstract
Keywords
- research
- manuscript
- publishing
Often, in personal conversations, we hear several reasons including time, resources, and usefulness of published reports and of research itself for the lack of interest by clinicians to publish scientific manuscripts. The number of patients treated is considered a more useful indicator of clinical care acumen than the number of scientific manuscripts published. I argue that publishing a scientific manuscript that results from a research study is an integral part of clinical medicine and that lack of publishing renders clinical care incomplete.

\section{Introduction}

It is unknown when the first clinical research paper was published. One of the early scientific journals was the Philosophical Transactions of the Royal Society of London, which dates back to 1665 and is credited to Henry Oldenberg. ${ }^{1,2}$

Clinicians, especially those in low resource settings, primary care settings, and nonacademic settings, do not consider publishing scientific manuscripts as a priority or a necessity. There are several reasons why a clinician should publish. The quality of clinical care rests on a foundation of knowledge that we now call evidence. It is important to understand that knowledge is more than just evidence. It includes the generation, understanding, analysis, interpretation, and assimilation of evidence in the right context. It is inevitable that current standards of knowledge are continuously evaluated if clinical care has to progress. The knowledge that exists and generated needs to be compared, critiqued, and assimilated appropriately to improve clinical care. Improvements may mean discarding certain bits of existing practices or processes that are not useful, continuing with existing practices that are useful, or improving upon existing practices. Refuting existing evidence is not uncommon in the health care realm. ${ }^{3-6}$ Controversies regarding the quality of evidence, research methods, and utility of research are also not uncommon. ${ }^{7-12}$

Diseases and well-being are a result of an interplay of several factors including the individual, their environment, and genes, all of which are in a constant state of evolution. Diseases and their definitions, therefore, are also evolving. Scientific research is in a race with disease to keep pace with its ever-changing nature. If such scientific knowledge constantly evolves, how do we manage to keep ourselves in sync with it?

Medical knowledge, within the medical community and the public at large, can only be updated through regular communications. Within the medical field, communication occurs primarily through conferences, meetings, and a network of medical journals. It may also happen on social media discussion forums like Facebook, WhatsApp, and other similar media. These do have their own limitations. Conferences and meetings are not regular, maybe held in different distant locations, could be expensive to attend, and packed with busy schedules taxing one's attention such that one tends to forget one presentation when the next one starts.

Discussions are rare at conferences due to time constraints. Social media forums offer the scope for unlimited discussion but are usually informal, unstructured, and not necessarily backed by the appropriate use or levels of evidence. Discussion in social forums too is time consuming with plenty of viewpoints to filter.

Journals too have some limitations. Access that is restricted by high subscription fees is an issue that is increasingly resolved by the proliferation of open access journals. The quality of articles and the representation of different levels of health care may be an issue. However, one has to consider that journals can only choose from what is offered to them. 
Journals, however, have several major advantages compared with conferences and discussion forums. Journals, either in print or as digital archives, provide a ready reference for a clinician who faces a clinical dilemma. Clinicians, almost daily, face cases where they have to establish a precise diagnosis and prognosis, identify the optimal management strategy taking into account the context of the patient, and evaluate alternates. The clinician has to rely on a dependable source of information that has undergone stringent evaluation. Currently, the scientific journal is the source of such information. One can argue that textbooks are a better source but one must consider that textbooks rely on scientific journals for information, are dated, and, at best, are a summary of the wealth of information available in journals. Journals also offer scope for discussion through letters to the editors where readers can interact with the authors. Open review is also increasingly recognized where readers can view peer reviewer comments and models where every reader is a potential reviewer with a scope to post comments and ask for clarifications online.

So, returning to the query of, why should a clinician publish, I argue that a clinician who truly cares for their patients wants to provide the best possible care for the best possible outcomes for the patient. This is possible only by constantly updating one's knowledge through the reading of scientific communications and best practices, which currently reside in scientific journals. If one makes use of such knowledge shared by other peers, I argue that every clinician has a responsibility to share their knowledge as well with their peers. This can help to improve care for other patients at a much larger level. Additionally, sharing one's experience and knowledge with other clinicians allows for a larger evaluation of the utility of processes and is thus educative by itself.

Publishing a scientific article is a learning education process in itself. When well done, the research study is a learning process where one updates knowledge and evaluates certain aspects of that knowledge. Scientific manuscripts are peer-reviewed, which means they are evaluated for content by external independent judges or reviewers. The process of review is primarily meant to understand the science better, to understand the relevance and appropriateness of the results, and to ensure that research communications add value rather than noise to the existing knowledge. Good peer reviews are a constructive educative process often done on an equivalent footing with the reviewer and the researcher learning and educating each other. A manuscript that passes through this process lends itself to communication with other clinicians worldwide.

A clinician who publishes contributes to the education of their peers while learning at the same time and contributes to an improved clinical care worldwide. They are the ones who ensure that clinical care progresses. There are several other reasons to publish a manuscript which are important but of less relevance. These include the feel-good factor of having published, meeting mandatory requirements of a certain number of publications for career promotion, ego, reputation, and more such factors that lead to several gains from publishing beyond medical and scientific realms. Other reasons may include improved reputation as an expert leading to more referrals, intellectual pleasure, addresses including policy matters leading to an improved social interaction, more grants or funds and increased revenue, and the ability to attract high-quality people to work or collaborate which also helps to keep one on their toes.

I often hear clinicians say "I see so many patients every day and have treated so many of their illnesses. Why should I publish?" To them, I have only three questions:

"First, where is the evidence to say you have helped, and Second, were your results independently evaluated, and

Third, if you have helped so many, why are you not sharing

that knowledge and experience with a wider group so that more people can benefit?"

We also hear clinicians complain that they do not have the time to write scientific papers. In the course of a busy clinical practice, clinicians from a nonacademic setting may be hardpressed to find time which may take away from personal and family life. Pragmatically, it is not often possible to draw a clear line between personal and professional time in many settings. However, if we document each care in detail, we do not really need to take too much time to write up a manuscript. And needless to say, documenting each case in detail is also a reflection of the quality of care we provide and a measure of our transparency while providing that care.

One must admit that scientific manuscripts are often filled with technical jargon especially on the statistical front and may seem overwhelming and make one shy away from publishing. However, I feel that every manuscript does not need complex statistical maneuvering. A set of basic statistics can help to present most findings. The lack of statistical expertise should no longer be a reason to avoid publishing. Some scientific journal publishing houses suggest resources that can help you with several aspects of publishing. ${ }^{13}$

Another, understated worry, about publishing is the possibility of finding out that one does not really know as much as one thought one knew about a subject during the rejection of the article after peer review. Rejections and comments to improve are a part of the game. One can look at that as bruising to the ego or as ways to improve.

Writing a scientific manuscript is just a natural way to complete the clinical care process. As in clinical care, it involves meticulous planning, evaluation of alternates, assessing and analyzing results, and documenting findings. If you look at it carefully, every good clinician actually does research on every subject they manage. Good clinicians maintain good medical records with meticulous details about what has happened with each patient. A scientific manuscript can be seen as sharing these notes, in a slightly different format, through a more uniform language that is understood worldwide.

Should every clinician then publish, you may ask. I say why not? Every clinician has a clinical story to share that can help someone somewhere manage a patient better. Why not share that knowledge?

The study of medicine and care is constant. A good practitioner has a responsibility to be a learner as well as educator 
throughout their time in the field. The learner-educator interface is interlinked, dynamic, and constantly evolving. Do not publish so that you may not perish. Publish, so that you help your peers provide better care. Publish, so that patients receive better care.

\section{Disclosures}

None.

\section{References}

1 Leon LR Jr, Vargas HI. "Ghost" publications in residency applications from international medical graduates. J Am Coll Surg 2009;208(3):483-484, author reply 484

2 Breathneck CS. The golden heresy of truth. J Ir Coll Phys Surg 1995;24:114-121

3 Ioannidis JP, Haidich AB, Lau J. Any casualties in the clash of randomised and observational evidence? BMJ 2001;322(7291):879-880

4 Vandenbroucke JP. When are observational studies as credible as randomised trials? Lancet 2004;363(9422):1728-1731

5 Ioannidis JP, Ntzani EE, Trikalinos TA, Contopoulos-Ioannidis DG. Replication validity of genetic association studies. Nat Genet 2001;29(3):306-309
6 Lawlor DA, Davey Smith G, Kundu D, Bruckdorfer KR, Ebrahim S. Those confounded vitamins: what can we learn from the differences between observational versus randomised trial evidence? Lancet 2004;363(9422):1724-1727

7 Chalmers I, Glasziou P. Avoidable waste in the production and reporting of research evidence. Lancet 2009;374(9683):86-89

8 Stuckler D, King L, Robinson H, McKee M. WHO's budgetary allocations and burden of disease: a comparative analysis. Lancet 2008;372(9649):1563-1569

9 Perel P, Miranda JJ, Ortiz Z, Casas JP. Relation between the global burden of disease and randomized clinical trials conducted in Latin America published in the five leading medical journals. PLoS One 2008;3(2):e1696

10 Tallon D, Chard J, Dieppe P. Relation between agendas of the research community and the research consumer. Lancet 2000;355(9220):2037-2040

11 Hewitt C, Hahn S, Torgerson DJ, Watson J, Bland JM. Adequacy and reporting of allocation concealment: review of recent trials published in four general medical journals. BMJ 2005;330(7499):1057-1058

12 Rutjes AW, Reitsma JB, Di Nisio M, Smidt N, van Rijn JC, Bossuyt PM. Evidence of bias and variation in diagnostic accuracy studies. CMAJ 2006;174(4):469-476

13 Author Services. Available at: https://www.elsevier.com/ en-in/authors/author-services. Accessed July 8, 2017 